\title{
Article \\ Performance Analysis of Intelligent Reflecting Surface-Assisted Multi-Users Communication Networks
}

\author{
Muhamad Mustaghfirin ${ }^{1}$, Keshav Singh $\left.{ }^{1, *} \mathbb{(}\right)$, Sudip Biswas ${ }^{2}\left(\mathbb{D}\right.$ and Wan-Jen Huang ${ }^{1}$ \\ 1 Institute of Communications Engineering, National Sun Yat-sen University, Kaohsiung 80424, Taiwan; \\ muhamad.mustaghfirin@g-mail.nsysu.edu.tw (M.M.); wanjen.huang@g-mail.nsysu.edu.tw (W.-J.H.) \\ 2 Department of Electronics and Communications Engineering, Indian Institute of Information Technology, \\ Guwahati 781015, India; sudip.biswas@ieee.org \\ * Correspondence: keshav.singh@mail.nsysu.edu.tw
}

Citation: Mustaghfirin, M.; Singh, K. Biswas, S.; Huang, W.-J. Performance Analysis of Intelligent Reflecting Surface-Assisted Multi-Users Communication Networks.

Electronics 2021, 10, 2084. https:// doi.org/10.3390/electronics10172084

Academic Editor: Christos J. Bouras

Received: 16 July 2021

Accepted: 24 August 2021

Published: 27 August 2021

Publisher's Note: MDPI stays neutral with regard to jurisdictional claims in published maps and institutional affiliations.

Copyright: (c) 2021 by the authors. Licensee MDPI, Basel, Switzerland. This article is an open access article distributed under the terms and conditions of the Creative Commons Attribution (CC BY) license (https:// creativecommons.org/licenses/by/ $4.0 /)$.

\begin{abstract}
An intelligent reflecting surface (IRS) is an array that consists of a large number of passive reflecting elements. Such a device possesses the potential to extend the coverage of transmission in future communication networks by overcoming the effects of non line-of-sight propagation. Accordingly, to present the case for utilizing IRS panels in future wireless networks, in this paper, we analyze a multi-user downlink network aided by IRS. In particular, by using a realistic 5G channel model, we compare the performance of the IRS-aided network with a decode and forward (DF) relay-aided scenario and a network without IRS or relay. Our analysis revealed the following: (i) At best, communication aided by a DF relay with perfect channel state information (CSI) could match the performance of the IRS-aided network with imperfect CSI when the channel estimation error was high and the number of users was large. (ii) IRS-aided communication outright outperformed the DF relay case when the transmit power was high or the number of users in the network was low. (iii) Increasing the number of elements in an IRS translated to greater quality of service for the users. (iv) IRS-aided communication showed better energy efficiency compared with the other two scenarios for higher quality of service requirements.
\end{abstract}

Keywords: intelligent reflecting surface; multi-users communications; energy-efficiency; powerminimization

\section{Introduction}

The rapid surge in global mobile data traffic has accentuated the deployment of $5 \mathrm{G}$ communications in several parts of the world. Based on a recent prediction of the International Telecommunication Union (ITU), it can be assumed that this trend will continue to grow exponentially until 2030 [1]. In particular, as per the ITU, the overall mobile data traffic is expected to reach an astonishing 5 zettabytes (ZB) per month globally. While 5G is expected to cater to some of these needs, it is almost certain that $5 \mathrm{G}$ will reach its limits before 2030. Accordingly, researchers have started to make inroads towards analyzing and developing the next-generation mobile communication technology.

Recently, there has been a great deal of interest from both industry and academia in an evolving hardware technology called intelligent reflecting surface (IRS) [2,3]. An IRS is a two-dimensional metasurface with reconfigurable properties [4]. In particular, the reflection, scattering, and diffraction properties of the array of passive discrete elements of an IRS can be controlled using software. It can be noted that the IRS is neither part of the transmitter radio nor the receiver radio. It is a novel way to control the wireless propagation medium, which, until now, had been deemed as uncontrollable.

Accordingly, the fundamental role of an IRS is to affect the dispersion of wireless signals transmitted by other devices, without producing its own signals. It can be used in a wireless network to assist communication by generating supplementary propagation paths, enhancing the characteristics of existing paths, and alleviating interference [5]. 
Related work on IRS-aided communication specifically centered on rate analysis has been carried out by [6,7]. In particular, the authors in [6] proposed a spectrum sharing solution to cater to large data demands owing to a large number of users in indoor environments. Next, using IRS, the authors in [8] achieved the same rate performance as a benchmark massive multiple-input multiple-output (MIMO) system with a significantly lower number of active antennas. In [9], the error-rate performance was improved by designing beamformers, such that the direct link signal constructively added at the user-side.

By providing an approximation of the achievable data rate, the authors in [10] analyzed the performance of IRS and derived the required phase shifts of the IRS panels under a data-rate degradation constraint. Similarly, in [11], it was shown that an IRS-based system could achieve excellent sum-rate performance by using a moderate number of IRS elements and a diminutive number of discrete phase shifts. Last but not least, in [7], the authors optimized the transmit power of IRS-aided communication to maximize the sum rate of the system.

On a similar note, current communication systems employ decode and forward (DF) relays to extend coverage in wireless networks [12]. However, the fundamental distinction of an IRS with a DF relay is that the relay actively processes the received signal before retransmitting the decoded signal with a certain power, while an IRS simply reflects the signal passively through beamforming without attempting to decode the signal. With regard to performance comparison between IRS and relay, the authors in [13] analyzed the impact of 5G channel models on IRS-aided communication and, hence, compared it with a DF relay for single user communication.

Next, in [14], the authors compared IRS with a DF relay in terms of the rate and energy-efficiency for a single-input single-output (SISO) system. In particular, the authors stated that IRS achieved better energy efficiency compared with the relay for higher rate requirements. Similarly, the authors in $[15,16]$ compared the energy-efficiency performance of an IRS-aided multi-user communication network with that of the AF relaying network. Both works testified that IRS provided more efficient communication than relays.

Based on the above, this paper analyzes the potential for using IRS in a multi-user 5G communication network. In particular, by using 5G channel models defined by 3GPP [17], we strive to obtain a reasonable correlation between IRS-supported communication and DF relay-assisted transmission for both the cases of perfect and imperfect channel-stateinformation (CSI).

We also analyze the effect of an increasing number of users on the performance of both systems, while we devise the size of IRS array required to better the performance of conventional relaying. Further, the problem of transmit power minimization with constraints on quality of service (QoS) and energy-efficiency (EE) maximization with constraints on QoS are considered, whereby a holistic comparison of the IRS assisted network with respect to the DF-relay-based communication is presented. In a nutshell, we recognize scenarios where IRSs can coexist with classic relaying in a future beyond $5 \mathrm{G}$ wireless networks.

Notation: This paper uses the following notations. $x$ represents a scalar, $\mathbf{x}$ represents a vector, and $\mathbf{X}$ represents a matrix. The notation $x \sim \mathcal{N}_{\mathbb{C}}\left(0, \sigma^{2}\right)$ denotes that the random variable $x$ is a complex circularly symmetric Gaussian with zero mean and variance $\sigma^{2}$. For any vector $\mathbf{v}, v_{i}$ is the $i$-th element of $\mathbf{v}$. $\mathbb{R}$ and $\mathbb{C}$ denote real and complex number sets, respectively, and $j \triangleq \sqrt{-1}$ is the imaginary unit.

\section{System Model and Problem Statement}

We consider a multi-user (MU) downlink communication scenario involving a basestation (BS) and $K$ multiple users. For the sake of analytical tractability, we assume that the $\mathrm{BS}$ and users are all equipped with single antennas. (Note that the multiple-antenna case will be considered in future extensions of this work). The BS communicates with the user $k$ through a deterministic flat fading channel represented by $h_{\mathrm{sd}, k} \in \mathbb{C}$. We term this form 
of communication as basic MU communication. Then, the signal received at the user $k$ is expressed as

$$
y_{k}=h_{\mathrm{sd}, k} \sqrt{p_{k}} s+w_{k}
$$

where $p$ is the transmit power, $s$ is the unit power information signal, and $w_{k} \sim \mathcal{N}_{\mathbb{C}}\left(0, \sigma^{2}\right)$ denotes the additive white Gaussian noise at the user $k$ with zero mean and variance $\sigma^{2}$. Since the BS broadcasts the same signal to all users in a certain location, the inter-user interference is neglected. The antenna gains at the transmitter and receiver are incorporated into the channel for simplification of the notation similar to [14]. Accordingly, the achievable rate at the $k$-th user can be given as

$$
R_{\mathrm{BASIC}, k}=\log _{2}\left(1+\frac{p_{k}\left|h_{\mathrm{sd}, k}\right|^{2}}{\sigma^{2}}\right) .
$$

However, when the signal passes through a blockage, such as buildings, trees, etc., (defined as NLoS), the achievable rate of the user decreases drastically. In this case, the use of IRS or relay can maintain the channel capacity by compensating for the NLoS effects and accordingly enhance the coverage of a communication network.

\subsection{IRS-Assisted MU Communications}

In this scenario, the BS sends the signal to $K$ users in the presence of blockages through the aid of an IRS as depicted in Figure 1. The IRS is equipped with $N$ passive reflecting elements. The signals reflected by each element of the IRS and the signals transmitted by the source can be constructively summed at the destination. Accordingly, the received signal at user $k$, with $k=1,2,3, \ldots, K$, is expressed as

$$
y_{k}=\left(h_{\mathrm{sd}, k}+\mathbf{h}_{\mathrm{sr}}^{T} \boldsymbol{\Theta} \mathbf{h}_{\mathrm{rd}, k}\right) \sqrt{p_{k}} s+w_{k}
$$

where $h_{\mathrm{sd}, k} \in \mathbb{C}, \mathbf{h}_{\mathrm{sr}} \in \mathbb{C}^{N}$, and $\mathbf{h}_{\mathrm{rd}, k} \in \mathbb{C}^{N}$ denote the deterministic channels from the transmitter to the user $k$, from the transmitter to the IRS, and from the IRS to the user $k$, respectively. $\boldsymbol{\Theta}$ is the phase-shift matrix of the IRS, denoted as

$$
\boldsymbol{\Theta}=\alpha \operatorname{diag}\left(e^{j \theta_{1}}, \ldots, e^{j \theta_{n}}, \ldots, e^{j \theta_{N}}\right)
$$

Here, $\alpha \in(0,1]$ is the fixed amplitude reflection coefficient and $\theta_{n}$ is the phase of the $n$th reflecting element of the IRS, which can be optimized. Hence, the channel capacity of the IRS assisted network is given as follows

$$
\begin{aligned}
R_{\mathrm{IRS}}(N) & =\max _{\theta_{1}, \ldots, \theta_{N}} \log _{2}\left(1+\frac{p\left|h_{\mathrm{sd}}+\mathbf{h}_{\mathrm{sr}}^{\mathrm{T}} \mathbf{\Theta} \mathbf{h}_{\mathrm{rd}}\right|^{2}}{\sigma^{2}}\right) \\
& =\log _{2}\left(1+\frac{p\left(\left|\mathbf{h}_{\mathrm{sd}}\right|+\alpha \sum_{n=1}^{N}\left|\left[\mathbf{h}_{\mathrm{sr}}\right]_{n}\left[\mathbf{h}_{\mathrm{rd}}\right]_{n}\right|\right)^{2}}{\sigma^{2}}\right)
\end{aligned}
$$

Accordingly, the achievable rate of the $k$-th user can be represented as

$$
R_{\mathrm{IRS}, k}(N)=\log _{2}\left(1+\frac{p_{k}\left(\left|h_{\mathrm{sd}, k}\right|+N \alpha\left|h_{\mathrm{sr}}\right|\left|h_{\mathrm{rd}, k}\right|\right)^{2}}{\sigma^{2}}\right) .
$$

The expression (7) can be obtained for any given $\Theta$. Note that

$$
\mathbf{h}_{\mathrm{sr}}^{\mathrm{T}} \boldsymbol{\Theta} \mathbf{h}_{\mathrm{rd}}=\alpha \sum_{n=1}^{N} \mid\left[\mathbf{h}_{\mathrm{sr}}\right]_{n}\left[\mathbf{h}_{\mathrm{rd}}\right]_{n} .
$$


Therefore, the maximum capacity or achievable rate can be obtained when the IRS phase shifts are picked as $\theta_{n}=\arg \left(\mathbf{h}_{\mathrm{sd}}\right)-\arg \left(\left[\mathbf{h}_{\mathrm{sr}}\right]_{n}\left[\mathbf{h}_{\mathrm{rd}}\right]_{n}\right)$ in order to make every term in the sum have the same phase as $\mathbf{h}_{\text {sd }}$ ([18], Section III-B).

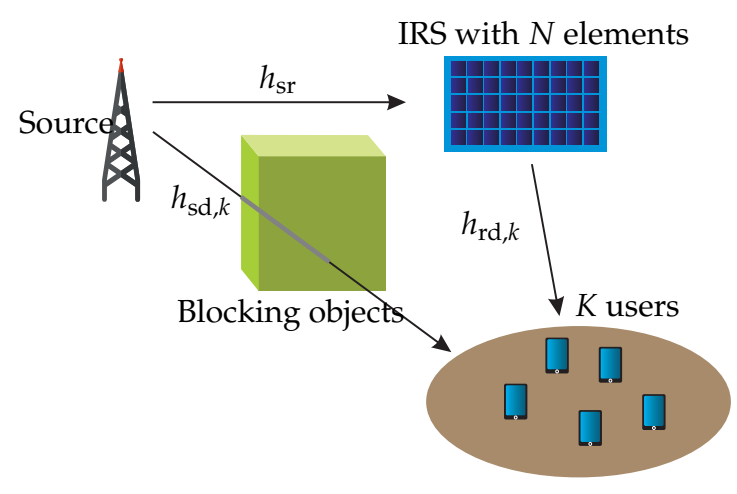

Figure 1. IRS-assisted multi-user communications.

\subsection{Relay-Assisted MU Communications}

In this scenario, we employ a DF relay instead of the IRS to extend coverage as illustrated in Figure 2. The signal transmission from the source to the destination is divided into two stages. The first stage involves the communication between the BS and the user. Accordingly, the received signal at user $k$ can be given as

$$
y_{\mathrm{d}, k}=h_{\mathrm{sd}, k} \sqrt{p_{1}} s+w_{1 \mathrm{~d}, k}
$$

where $p_{1}$ is the transmit power, $s$ is the unit information signal, and $w_{1 \mathrm{~d}, k} \sim \mathcal{N}_{\mathbb{C}}\left(0, \sigma^{2}\right)$ is the noise at the user $k$. In the same stage, the relay will also receive the signal that is given as

$$
y_{1 \mathrm{r}}=g_{\mathrm{sr}} \sqrt{p_{1}} s+w_{1 \mathrm{r}}
$$

where $g_{\text {sr }} \in \mathbb{C}$ is the channel between the BS and relay and $w_{1 \mathrm{r}} \sim \mathcal{N}_{\mathbb{C}}\left(0, \sigma^{2}\right)$ is the noise at the relay.

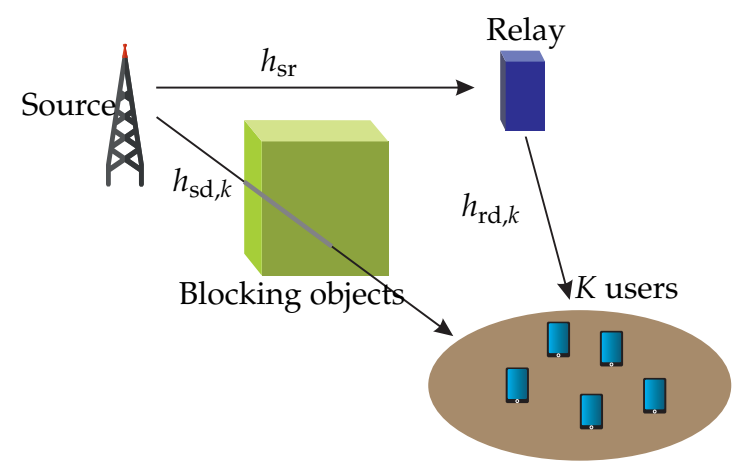

Figure 2. Relay-assisted multi-user communications.

In the second stage, the relay decodes, re-encodes, and then sends the signal to the user with the power transmit $p_{2}$. Accordingly, the received signal at the user $k$ can be given as

$$
y_{2 \mathrm{~d}, k}=g_{\mathrm{rd}, k} \sqrt{p_{2}} s+w_{2 \mathrm{~d}, k}
$$

where $g_{\mathrm{rd}, k} \in \mathbb{C}$ is the channel between the relay and the user $k$ and $w_{1 \mathrm{~d}, k} \sim \mathcal{N}_{\mathbb{C}}\left(0, \sigma^{2}\right)$ is the noise at the user $k$. Now, the received signal in (9) and (11) can be constructively 
combined using the maximum ratio combining (MRC) [14], such that the achievable rate at the user $k$ is given as

$$
R_{\mathrm{DF}, k}=\frac{1}{2} \log _{2}\left(1+\min \left(\frac{p_{1}\left|g_{\mathrm{sr}}\right|^{2}}{\sigma^{2}}, \frac{p_{1}\left|h_{\mathrm{sd}, k}\right|^{2}}{\sigma^{2}}+\frac{p_{2}\left|g_{\mathrm{rd}, k}\right|^{2}}{\sigma^{2}}\right)\right) .
$$

Here, $p_{1}$ and $p_{2}$ are the transmit powers in the first and second stage, respectively.

Now, in order to obtain a fair comparison with respect to the IRS scenario, we set $p_{1}$ and $p_{2}$ optimally similar to [14] for the DF relay case. When $\left|h_{\mathrm{sd}, k}\right|^{2}>\left|g_{\mathrm{sr}}\right|^{2}$,

$$
\min \left(\frac{p_{1}\left|g_{\mathrm{sr}}\right|^{2}}{\sigma^{2}}, \frac{p_{1}\left|h_{\mathrm{sd}, k}\right|^{2}}{\sigma^{2}}+\frac{p_{2}\left|g_{\mathrm{rd}, k}\right|^{2}}{\sigma^{2}}\right)=\frac{p_{1}\left|g_{\mathrm{sr}}\right|^{2}}{\sigma^{2}} .
$$

The above is maximized by setting $p_{1}=2 p$ and $p_{2}=0$, which results in the relay not being not implemented at all since this condition makes $R_{\mathrm{BASIC}}>R_{\mathrm{DF}}$. Conversely, when $\left|h_{\mathrm{sd}, k}\right|^{2} \leq\left|g_{\mathrm{sr}}\right|^{2}, R_{\mathrm{DF}}$ is maximized by assigning $p_{1}$ and $p_{2}$ to attain

$$
\frac{p_{1}\left|g_{\mathrm{sr}}\right|^{2}}{\sigma^{2}}=\frac{p_{1}\left|h_{\mathrm{sd}, k}\right|^{2}}{\sigma^{2}}+\frac{p_{2}\left|g_{\mathrm{rd}, k}\right|^{2}}{\sigma^{2}}
$$

under the constraint $p=\frac{p_{1}+p_{2}}{2}$. Hence, the relay-assisted communication needs to choose between two conditions depending of $\left|h_{\mathrm{sd}, k}\right|^{2}$ and $\left|g_{\mathrm{sr}}\right|^{2}$ in such the achievable rate at the user $k$ in (12) redefine in (15).

$$
R_{\mathrm{DF}, k}= \begin{cases}\frac{1}{2} \log _{2}\left(\frac{p_{k}\left|h_{\mathrm{sd}, k}\right|^{2}}{\sigma^{2}}\right) & \text { if }\left|h_{\mathrm{sd}, k}\right|^{2}>\left|g_{\mathrm{sr}}\right|^{2} \text { for } \forall k, \\ \frac{1}{2} \log _{2}\left(\frac{2 p_{k}\left|g_{\mathrm{rd}, k}\right|^{2}\left|g_{\mathrm{sr}}\right|^{2}}{\left(\left|g_{\mathrm{sr}}\right|^{2}+\left|g_{\mathrm{rd}, k}\right|^{2}-\left|h_{\mathrm{sd}, k}\right|^{2}\right) \sigma^{2}}\right) & \text { if }\left|h_{\mathrm{sd}, k}\right|^{2} \leq\left|g_{\mathrm{sr}}\right|^{2} \text { for } \forall k .\end{cases}
$$

\section{Analytical Performance Comparison}

In this section, we analyze the performance among the IRS, and relay aided MU communications. As a benchmark for comparison, we also present the basic MU communication scenario without the aid of IRS/relay.

\subsection{Transmit Power for Given QoS}

Let us consider the scenario when the minimum QoS requirement of the network in terms of the data rate is $\bar{R}$. Accordingly, the task is to calculate the power required at the BS to transmit the signal. Accordingly, the transmit power can be calculated using Equations (2), (7) and (12) in a way similar to ([14], Corollary). For the required data rate $\bar{R}$, the basic MU case will require a transmit power given as

$$
p_{\mathrm{BASIC}, k}=\left(2^{\bar{R}}-1\right) \frac{\sigma^{2}}{\left|h_{\mathrm{sd}, k}\right|^{2}},
$$

Similarly, the IRS-assisted communication needs a transmit power given as

$$
p_{\mathrm{IRS}, k}(N)=\left(2^{\bar{R}}-1\right) \frac{\sigma^{2}}{\left(\left|h_{\mathrm{sd}, k}\right|+N \alpha \sqrt{\left|h_{\mathrm{sr}}\right| \mid h_{\mathrm{rd}, k}}\right)^{2}}
$$

and the DF-relay-assisted communication needs a transmit power, which is given as

$$
p_{\mathrm{DF}, k}= \begin{cases}\left(2^{2 \bar{R}}-1\right) \frac{\sigma^{2}}{\left|h_{\mathrm{sd}, k}\right|^{2}} & \text { if }\left|h_{\mathrm{sd}, k}\right|>\left|g_{\mathrm{sr}}\right| \text { for } \forall k, \\ \left(2^{2 \bar{R}}-1\right) \frac{\left(\left|g_{\mathrm{sr}}\right|^{2}+\left|g_{\mathrm{rd}, k}\right|^{2}-\left|h_{\mathrm{sd}, k}\right|^{2}\right) \sigma^{2}}{2\left|g_{\mathrm{rd}, k}\right|^{2}\left|h_{\mathrm{sr}}\right|^{2}} & \text { if }\left|h_{\mathrm{sd}, k}\right| \leq\left|g_{\mathrm{sr}}\right| \text { for } \forall k .\end{cases}
$$




\subsection{Energy Efficiency}

The energy efficiency (EE) of the network is defined as $B \cdot \bar{R} / P_{\text {total }}$, where the total power $P_{\text {total }}$ includes both the transmit power and the dissipation at the hardware components of the system [14]. In the basic MU scenario, the total power consumption can be expressed as

$$
P_{\text {total }}^{\text {BASIC }}=\sum_{k=1}^{K} \frac{p_{\text {BASIC }, k}}{v}+P_{\mathrm{s}}+P_{\mathrm{d}}
$$

where $v \in(0,1]$ is the efficiency of the power amplifier, while $P_{\mathrm{s}}$ and $P_{\mathrm{d}}$ are the hardwaredissipated power at the source and destination, respectively.

When using IRS, the power consumption of the network can be formulated as

$$
P_{\text {total }}^{\mathrm{IRS}}(N)=\sum_{k=1}^{K} \frac{p_{\mathrm{IRS}, k}(N)}{v}+P_{\mathrm{s}}+P_{\mathrm{d}}+N \cdot P_{\mathrm{e}}
$$

where $P_{\mathrm{e}}$ is the power dissipation in each element of IRS affected by the process of adaptive phase-shifting. Now, for a given data rate requirement, the maximum energy efficiency in the IRS-assisted communication can be achieved by minimizing the total transmit power, which can be obtained by using the optimal number of IRS elements $N$ [14].

Proposition 1. Assume that both $\left|h_{\mathrm{sr}}\right|^{2}$ and $\left|h_{\mathrm{rd}}\right|^{2}$ are constants and independent of $N$. Then, the total power required in an IRS assisted communication to achieve a data rate of $\bar{R}$ is a convex function, which can be minimized using the optimal number of IRS elements obtained by $\frac{\partial}{\partial N} P_{\text {total }}^{\operatorname{IRS}}(N)=0$, and given as

$$
N^{\mathrm{opt}}=\sqrt[3]{\frac{\left(2^{\bar{R}}-1\right) \sigma^{2}}{\alpha^{2}\left|h_{\mathrm{sr}}\right|^{2}\left|h_{\mathrm{rd}}\right|^{2} P_{\mathrm{e}}}}-\frac{1}{\alpha} \sqrt{\frac{\left|h_{\mathrm{sd}}\right|^{2}}{\left|h_{\mathrm{sr}}\right|^{2}\left|h_{\mathrm{rd}}\right|^{2}}} .
$$

Proof of Proposition 1. Taking the second partial derivative of (20) with respect to $N$, we obtain

$$
\begin{aligned}
& \frac{\partial^{2}}{\partial^{2} N} P_{\text {total }}^{\text {IRS }}(N)=\frac{\partial^{2}}{\partial^{2} N} \frac{p_{\text {IRS }}(N)}{v}+P_{\mathrm{s}}+P_{\mathrm{d}}+N \cdot P_{\mathrm{e}} \\
& =\frac{1}{v} \frac{\partial^{2}}{\partial^{2} N} p_{\mathrm{IRS}}(N)+\frac{\partial^{2}}{\partial^{2} N} N \cdot P_{\mathrm{e}} \\
& =\frac{1}{v} \frac{\partial^{2}}{\partial^{2} N}\left(2^{\bar{R}}-1\right) \frac{\sigma^{2}}{\left(\left|h_{\mathrm{sd}}\right|+N \alpha \sqrt{\left|h_{\mathrm{sr}}\right| \mid h_{\mathrm{rd}}}\right)^{2}}+\frac{\partial^{2}}{\partial^{2} N} N \cdot P_{\mathrm{e}} \\
& =\frac{-2}{v} \frac{\partial}{\partial N}\left(2^{\bar{R}}-1\right) \frac{\sigma^{2} N}{\left(\left|h_{\mathrm{sd}}\right|+N \alpha \sqrt{\left|h_{\mathrm{sr}}\right| \mid h_{\mathrm{rd}}}\right)^{3}}+\frac{\partial}{\partial N} P_{\mathrm{e}} \\
& =\frac{6}{v}\left(2^{\bar{R}}-1\right) \frac{\sigma^{2} N^{2}}{\left(\left|h_{\mathrm{sd}}\right|+N \alpha \sqrt{\left|h_{\mathrm{sr}}\right| \mid h_{\mathrm{rd}}}\right)^{4}}>0 .
\end{aligned}
$$

From (22), we can observe that the function involving the total power consumed by an IRS-assisted communication in (20) is convex since $\frac{\partial^{2}}{\partial^{2} N} P_{\text {total }}^{\text {IRS }}(N)>0$ for any given data rate $\bar{R}>0$.

Similarly, for the DF relay scenario, the power consumption can be represented as

$$
P_{\text {total }}^{\mathrm{DF}}=\sum_{k=1}^{K} \frac{p_{\mathrm{DF}, k}}{v}+\frac{1}{2} P_{\mathrm{s}}+P_{\mathrm{d}}+P_{\mathrm{r}}
$$

where $P_{\mathrm{r}}$ is the hardware-dissipated power at the relay. 


\section{Numerical Results and Discussions}

\subsection{Channel Model}

\subsubsection{Perfect CSI}

Generally, it is a challenge to estimate the IRS channel due to the passiveness of IRS elements. Several works have been carried out to address the CSI estimation for IRS-aided wireless communication. Regarding IRS channel estimation, some methods have been investigated, such as the Brute Force Method [19], compressive sensing method [20], semi-passive method [21], and three phase channel estimation framework [22]. However, in this work, we adopted a method [23] where the source node and IRS are integrated through a central control unit (CCU) that is able to collect all the CSIs information.

We begin by considering an ideal scenario in which the CSI is perfectly known to the nodes in the network. We implement the 5G channel model as described in 3GPP TR 38.901 [17] and accordingly define the deterministic model for the channel gain using the definition of height and distance as illustrated in Figure 3. The concerned model is also considered in [24], which analyzes FR2 propagation in the range of 0.5-100 GHz.

For the simulation setup, we use fixed values for the height of the BS and the height of user, defined as $d_{\mathrm{BS}}=10 \mathrm{~m}$ and $d_{\mathrm{UE}}=1.5 \mathrm{~m}$, respectively. The value of $d_{2 \mathrm{D}}$ is varied depending on the distance between the BS and the users. The varying $d_{2 \mathrm{D}}$ determines the value of $d_{3 \mathrm{D}}$ with the relation $d_{3 \mathrm{D}}=\left(d_{\mathrm{BS}}^{2}+d_{2 \mathrm{D}}^{2}+\left(d_{\mathrm{BS}}-d_{\mathrm{UE}}\right)^{2}\right)^{\frac{1}{2}}$. We use a carrier frequency of $3 \mathrm{Ghz}$. Table 1 describes the path loss models for line-of-sight (LoS) and non-line-of-sight (NLoS) propagation. Now, we formulate the deterministic channel gain $\left(|h|^{2}\right.$ or $|g|^{2}$ ) denoted as the function of $d_{3 \mathrm{D}}$ by neglecting the shadowing effect as

$$
\left(|h|^{2} \text { or }|g|^{2}\right)\left(d_{3 \mathrm{D}}\right)[\mathrm{dB}]=G_{\mathrm{BS}}[\mathrm{dBi}]+\mathrm{G}_{\mathrm{UE}}[\mathrm{dBi}]+P L\left(d_{3 \mathrm{D}}\right) .
$$

Here, $G_{B S}$ and $G_{U E}$ are the antenna gains for the BS and the user equipment, respectively whereas the value of $P L\left(d_{3 \mathrm{D}}\right.$ can be obtained from the Table 1 depending on the propagation model (LoS or NLoS). The gain for this deterministic channel model is depicted in Figure 4 with respect to the distance $d_{3 \mathrm{D}}$.

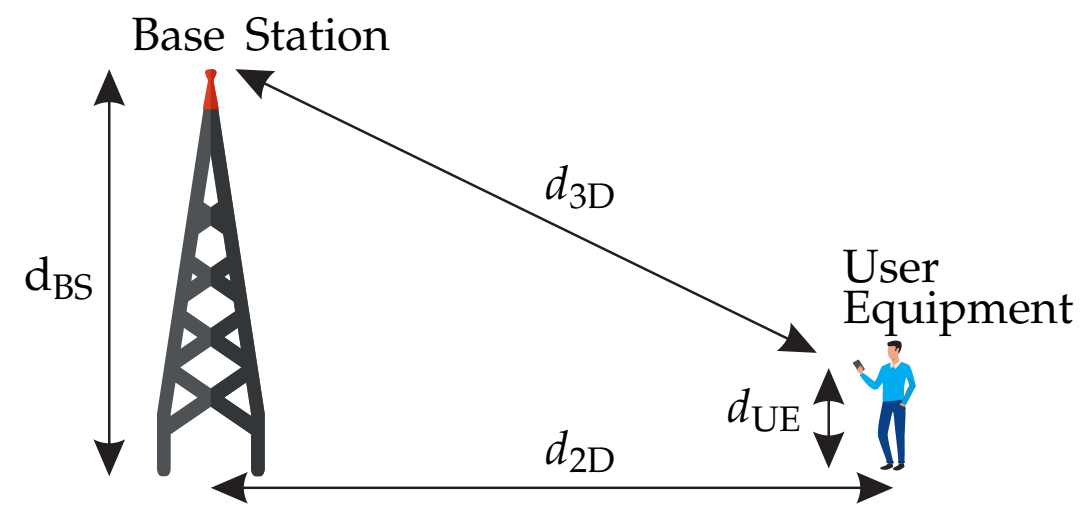

Figure 3. 3GPP model for Urban Micro (UMi) scenario, which includes the definition of height and distance. 


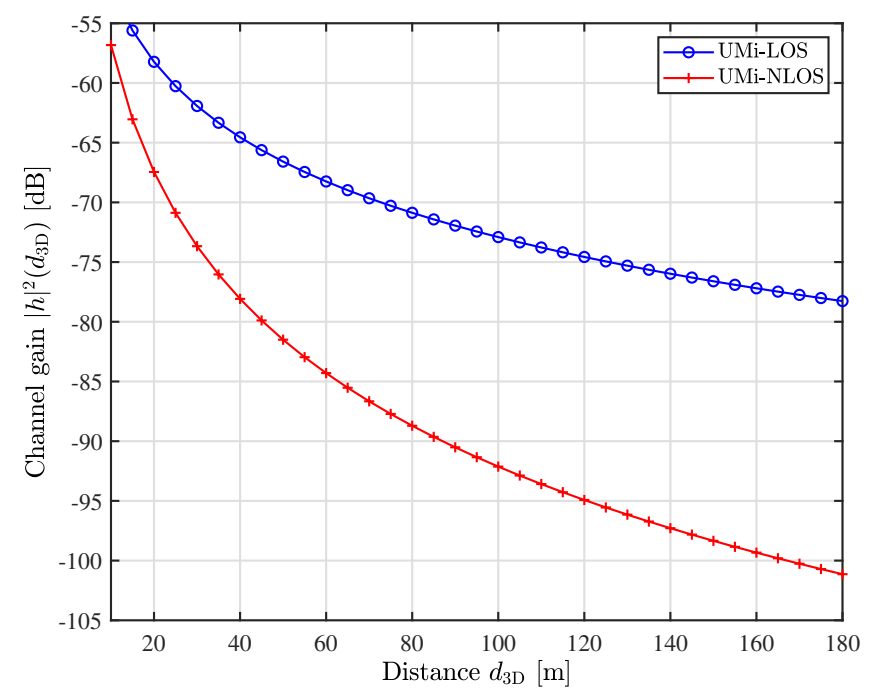

Figure 4. Channel gain $|h|^{2}$ with prefect CSI correspond to the distance $d_{3 \mathrm{D}}$ with the value $10 \mathrm{~m} \leq d_{3 \mathrm{D}} \leq 180 \mathrm{~m}$ in which the antenna gain $G_{\mathrm{BS}}$ and $G_{\mathrm{UE}}$ have been fixed to $8 \mathrm{dBi}$.

Table 1. Path loss models for urban micro cell (UMi) when $d_{2 \mathrm{D}}$ is less than the break point distance $d_{\mathrm{BP}}$. Distances are in meters, $f_{\mathrm{C}}$ is in $\mathrm{Hz}$, and $d_{\mathrm{BS}}=10 \mathrm{~m}$ ([17], Table 7.4.1-1).

\begin{tabular}{ll}
\hline & $P L_{\mathrm{LOS}}=32.4+21 \log _{10}\left(d_{3 \mathrm{D}}\right)+20 \log _{10}\left(f_{\mathrm{c}}\right)$, for $10 \mathrm{~m} \leq d_{2 \mathrm{D}} \leq d_{\mathrm{BP}}$ \\
& where $d_{\mathrm{BP}}=18 f_{\mathrm{c}} /\left(3 \times 10^{-8}\right) \mathrm{m}$ for $d_{\mathrm{UE}}=1.5 \mathrm{~m}$ and $d_{\mathrm{BS}}=10 \mathrm{~m}$ \\
\hline & $P L_{\mathrm{NLOS}}=\max \left(P L_{\mathrm{LOS}}, P L_{\mathrm{NLOS}}^{\prime}\right)$, where $P L_{\mathrm{NLOS}}^{\prime}=22.4+35.3 \log _{10}\left(d_{3 \mathrm{D}}\right)$ \\
$+21.3 \log _{10}\left(f_{\mathrm{c}}\right)-0.15$, for $d_{\mathrm{UT}}=1.5 \mathrm{~m}$ and $10 \mathrm{~m} \leq d_{2 \mathrm{D}} \leq 5 \mathrm{~km}$
\end{tabular}

\subsubsection{Imperfect CSI}

We also consider the scenario when the nodes in the network have imperfect CSI. Accordingly, the imperfect channels from source to destination, source to IRS, IRS to destination, source to relay, and relay to destination, which are denoted as $\hat{h}_{\mathrm{sd}}, \hat{h}_{\mathrm{sr}}$, $\hat{h}_{\text {rd }} \hat{g}_{\text {sr }}$, and $\hat{g}_{\text {rd }}$, respectively, can be given by [25]

$$
\begin{aligned}
& h_{\mathrm{sd}}=\hat{h}_{\mathrm{sd}}+\tilde{e}_{\mathrm{sd}} ; \\
& h_{\mathrm{sr}}=\hat{h}_{\mathrm{sr}}+\tilde{e}_{\mathrm{sr}} ; \\
& h_{\mathrm{rd}}=\hat{h}_{\mathrm{rd}}+\tilde{e}_{\mathrm{rd}} ; \\
& g_{\mathrm{sr}}=\hat{g}_{\mathrm{sr}}+\tilde{e}_{\mathrm{sr}} ; \text { and } \\
& g_{\mathrm{rd}}=\hat{g}_{\mathrm{rd}}+\tilde{e}_{\mathrm{rd}} .
\end{aligned}
$$

Here, $\tilde{e}_{\mathrm{sd}}, \tilde{e}_{\mathrm{sr}}$, and $\tilde{e}_{\mathrm{rd}}$ are the corresponding channel estimation errors modeled as i.i.d. and distributed as $\mathcal{N}_{\mathbb{C}}\left(0, \sigma_{\text {sd }}^{2}\right), \mathcal{N}_{\mathbb{C}}\left(0, \sigma_{\text {sr }}^{2}\right)$, and $\mathcal{N}_{\mathbb{C}}\left(0, \sigma_{\text {rd }}^{2}\right)$, respectively. For brevity, we set $\tilde{e}_{\mathrm{sd}}=\tilde{e}_{\mathrm{sr}}=\tilde{e}_{\mathrm{rd}}=\tilde{e}$.

\subsection{Simulation Setup}

IRS panels/relay nodes are deployed at a fixed distance, $d_{\mathrm{sr}}=80 \mathrm{~m}$ from the BS on the top of a building with height $d_{R}=10 \mathrm{~m}$. Since there are several blockages between the propagation path of the BS and the UEs as illustrated in Figure 5, there exist only NLoS paths between the BS and UEs. This is the primary use case scenario of deploying IRS or relay, whereby LoS channels can be carved out between the BS and UEs. 
Now, the channel gains with respect to the distance $d_{3 \mathrm{D}}$ can be calculated using Equation (24). We define the antenna gain at the BS and IRS/ relay with the same value of $8 \mathrm{dBi}$, while the UEs are assumed to be equipped with omni-directional antennas with gain $0 \mathrm{dBi}$. The number of elements for the IRS is set to $N=250$, $\alpha=1$, and the source transmit power is set at $p=20 \mathrm{dBm}$. The transmission bandwidth is $B=10 \mathrm{MHz}$ with the corresponding noise power set at $-94 \mathrm{dBm}$.

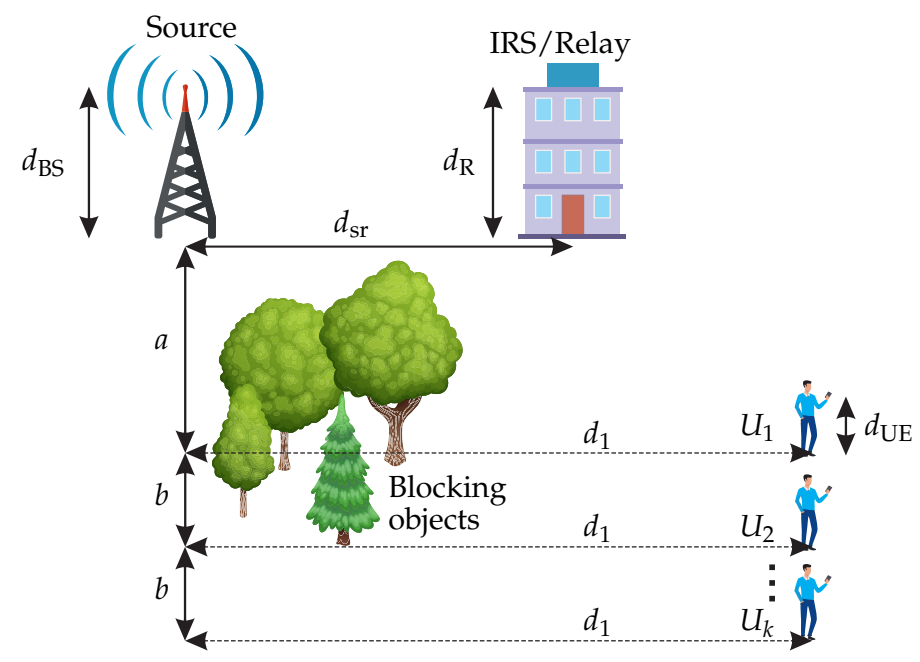

Figure 5. The simulation scenario where $d_{1}$ is varied with the same value for all users, while $a=10 \mathrm{~m}$ is the closest user distance from the source and $b=1 \mathrm{~m}$ are the distances among users.

Experiments are performed for three scenarios: (i) downlink communication between BS and users without IRS/ relay (basic MU communication), (ii) downlink communication between BS and users with IRS, and (iii) downlink communication between BS and users with relay. The achievable rates for scenarios i, ii, and iii are calculated using Equations (2), (7) and (12), respectively. Accordingly, Figure 6 depicts the achievable rate obtained in case i, IRS-aided, and relay-aided multi-users communication scenarios for varying the number of UEs for both perfect and imperfect CSI considerations.

It can be seen from Figure $6 \mathrm{a}$ ( $\tilde{e}=0.2$ for imperfect CSI case) that, under a perfect CSI, the IRS-aided communication achieved the highest rate followed by the DF relayaided system, which outperformed the basic MU communication without the aid of either of them. Further, as expected, the achievable rate decreased with the increasing number of users for the three scenarios. However, the IRS-aided communication fared better than the rest. In particular, a 250-element IRS could provide higher achievable rates to 100 users than a DF relay or a set-up without either of them. Figure $6 \mathrm{~b}$ shows similar trends but for $\tilde{e}=0.5$. Accordingly, at best, the DF relay with perfect CSI could match the performance of IRS with imperfect CSI when the channel estimation error was high and the number of users was large. 


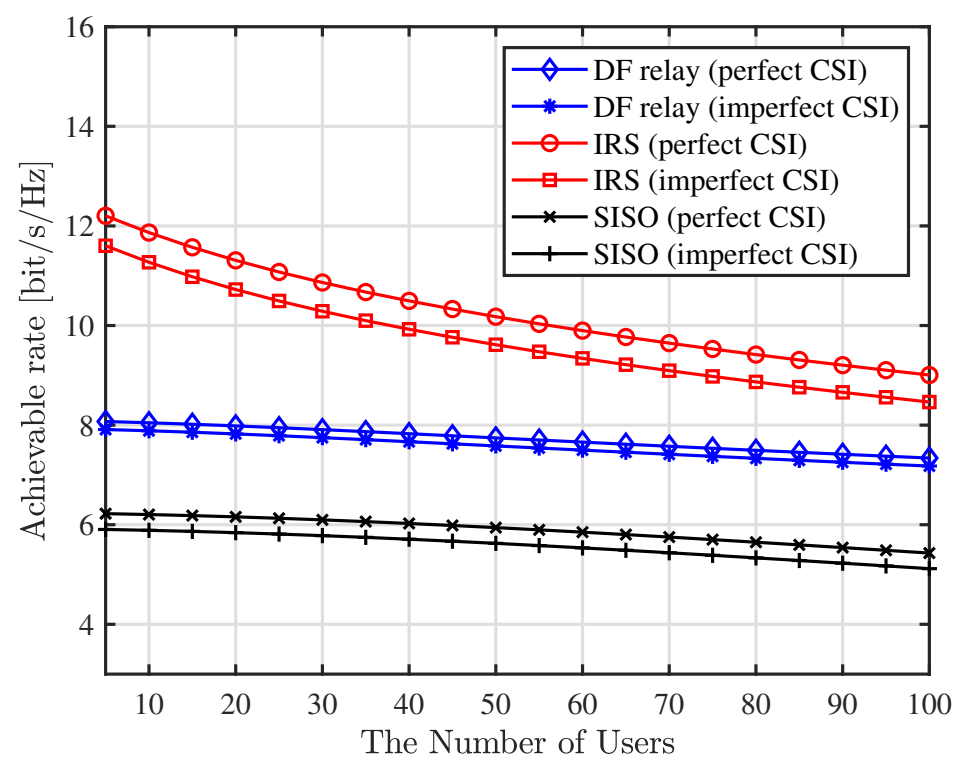

(a) Estimated channel error $\tilde{e}=0.2$.

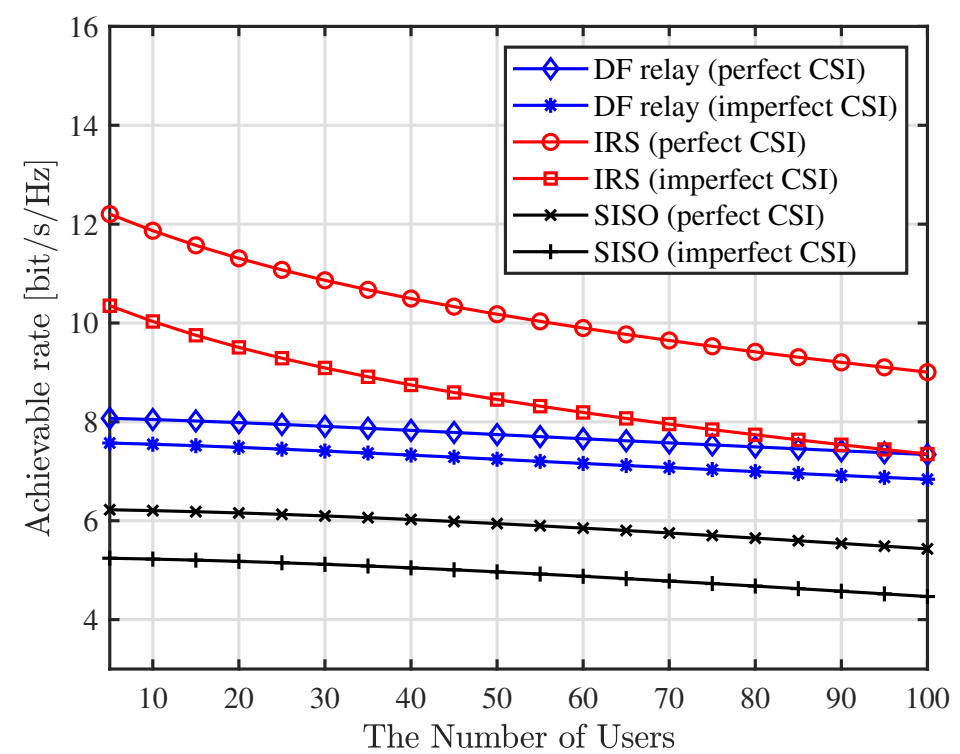

(b) Estimated channel error $\tilde{e}=0.5$.

Figure 6. The achievable rate for different number of users where $d_{1}$ and $d_{\text {sr }}$ are set to fixed values of $100 \mathrm{~m}$ and $80 \mathrm{~m}$, respectively, in terms of perfect and imperfect CSI.

Next, in Figure 7, we study the effect of transmit power for the perfect CSI case on the achievable rate and two different considerations of the total number of users. While, for the case with $K=10$ users, the IRS-aided communication outperformed the rest throughout the entire range of transmit power. For the case of $K=100$ users, a crossing point between the IRS and DF relay can be seen at $p=14 \mathrm{dBm}$. Accordingly it can be concluded that the IRS-aided communication outright outperformed the DF relay case when the transmit power was high or the number of users in the network was low. 


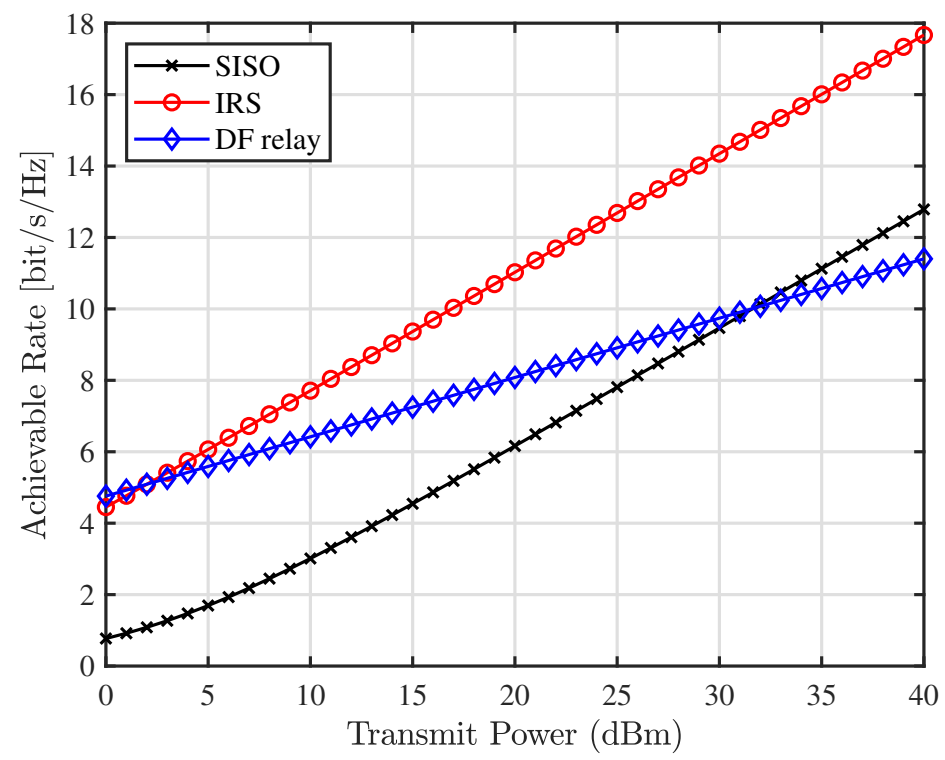

(a) $K=10$ users.

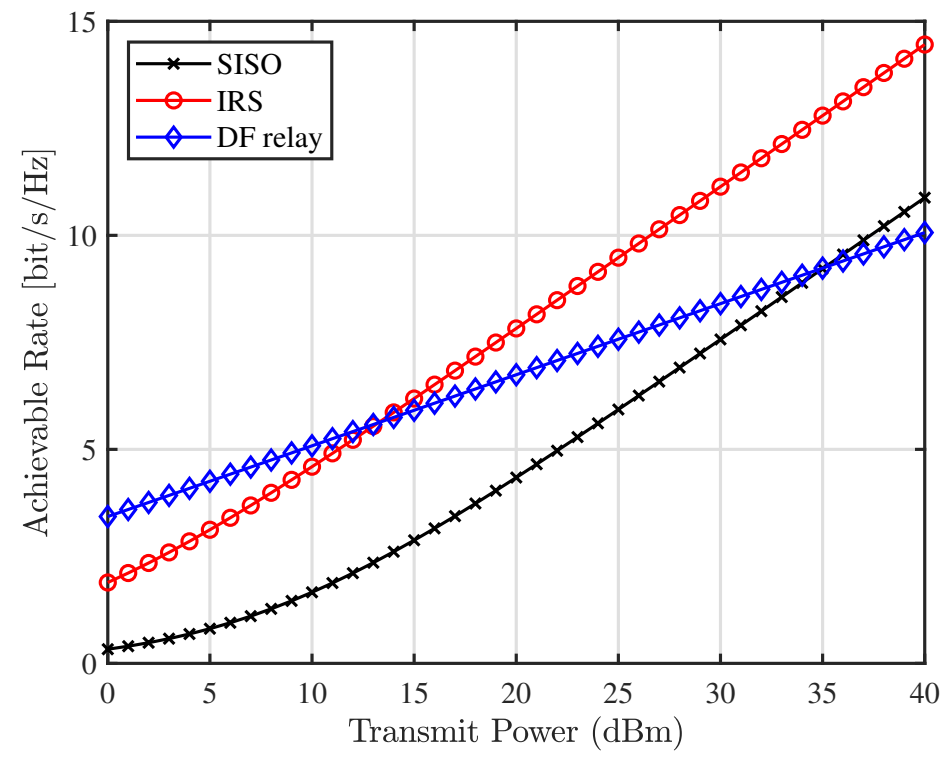

(b) $K=100$ users.

Figure 7. The achievable rate based on varying transmit power with different numbers of users.

Figure 8 shows the required transmit power with respect to distance $\left(d_{1}\right)$ for two data rate scenarios $\bar{R}=6 \mathrm{bit} / \mathrm{s} / \mathrm{Hz}$ and $\bar{R}=8 \mathrm{bit} / \mathrm{s} / \mathrm{Hz}$. Here, the number of users $K=100$. It can be seen from the figure that, under low data rate requirements $(\bar{R}=6 \mathrm{bit} / \mathrm{s} / \mathrm{Hz})$, the DF relay transmitted the least power among the three scenarios. However, when the data rate requirement increased to $\bar{R}=8 \mathrm{bit} / \mathrm{s} / \mathrm{Hz}$, the IRS- aided frameworks with 150 and 200 elements outperformed the rest. Accordingly, higher QoS requirements can be satisfied in wireless networks by using IRS panels, where the number of elements are greater than 150. 


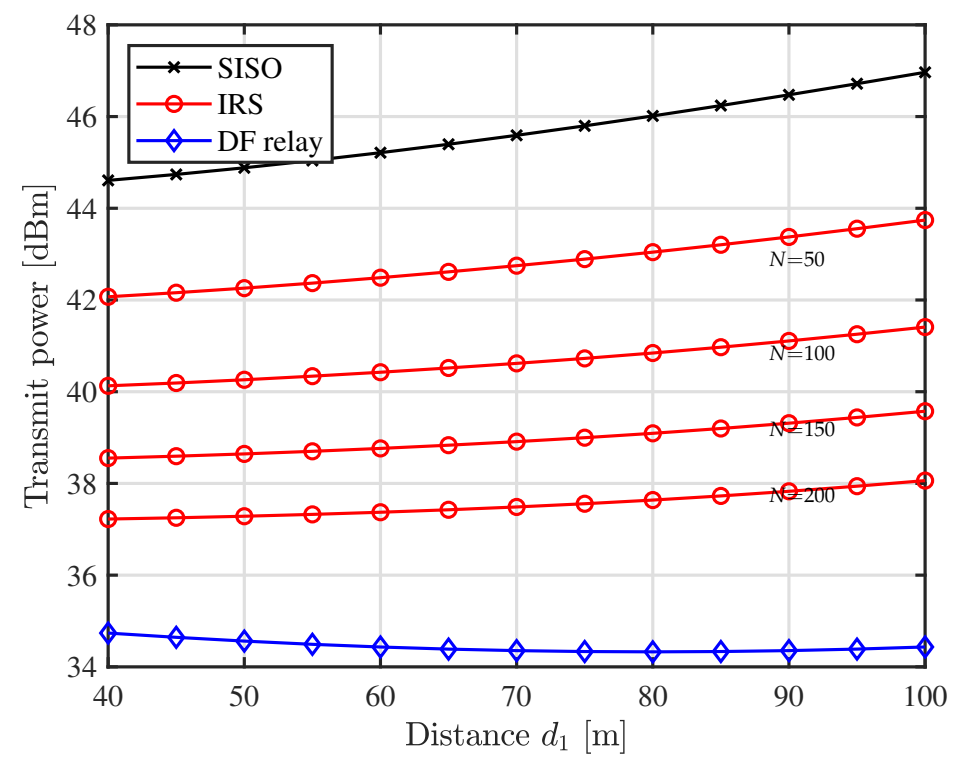

(a) $\bar{R}=6 \mathrm{bit} / \mathrm{s} / \mathrm{Hz}$.

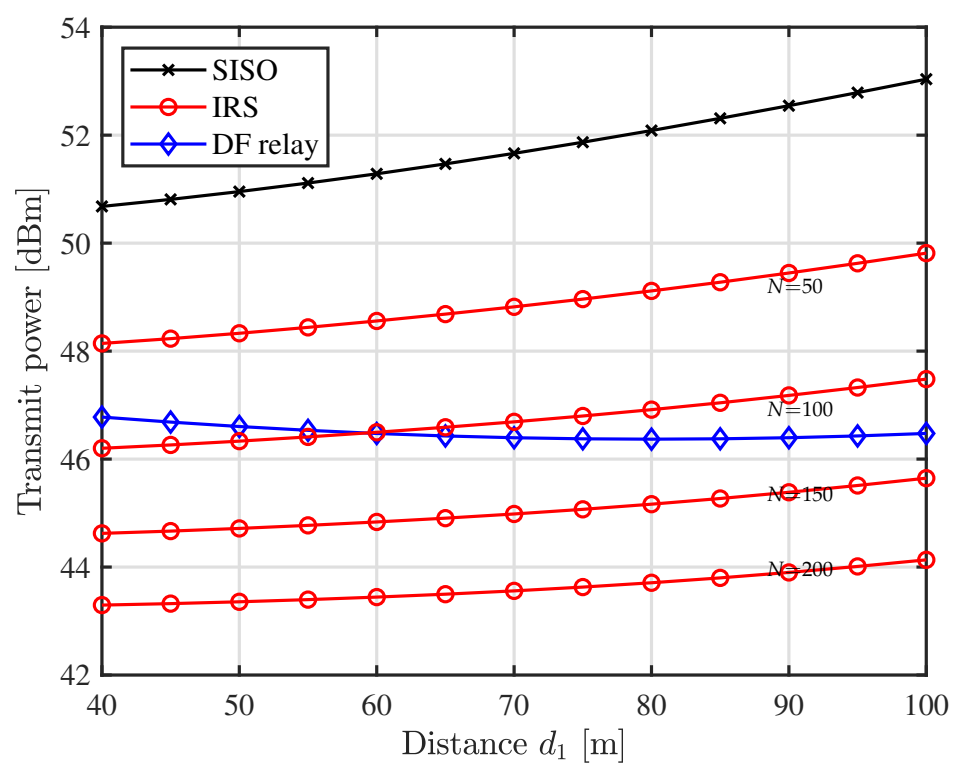

(b) $\bar{R}=8 \mathrm{bit} / \mathrm{s} / \mathrm{Hz}$.

Figure 8. The transmit power based on varying distance $d_{1}$ with the number of users $K=100$.

Additionally, in Figure 9, we analyze the energy-efficiency of the three scenarios for perfect CSI with respect to the achievable rate as discussed in Section 3. Here, $v=0.5, P_{\mathrm{s}}=P_{\mathrm{d}}=P_{\mathrm{r}}=100 \mathrm{~mW}$, and $P_{\mathrm{e}}=5 \mathrm{~mW}$ [15], $d_{1}=100 \mathrm{~m}$. We apply the optimal element number for IRS as derived in Equation (21) in order to find the maximum energy-efficiency. It can be seen from the figure that, although the peak energy-efficiency obtained for the DF relay case was higher than the scenario with IRS, the IRS-aided network maintained significant energy-efficiency levels throughout the entire range of the rate. Accordingly, it can be concluded that the IRS-aided communication showed better energy-efficiency compared with the other two scenarios for higher QoS requirements. 


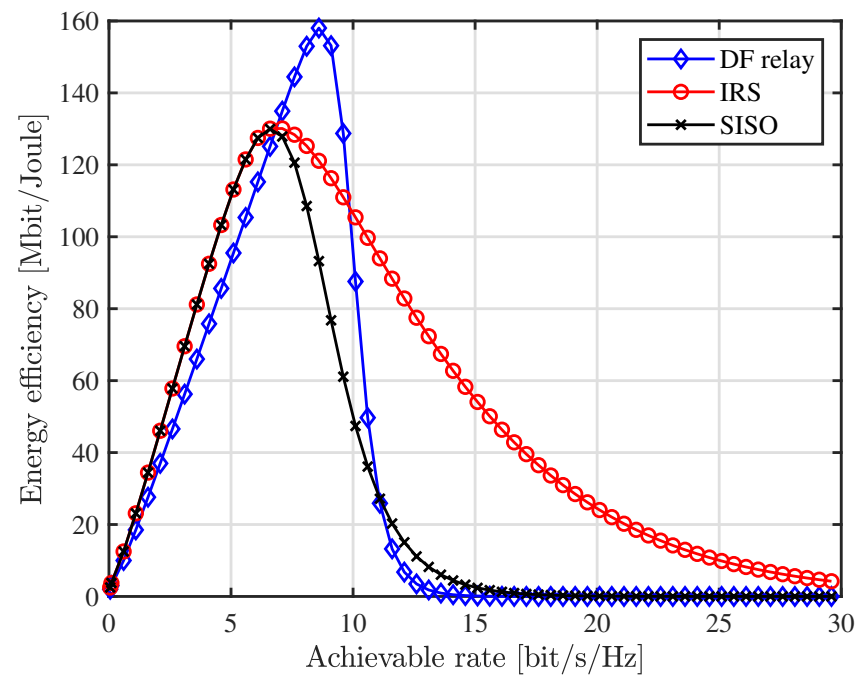

(a) $K=10$ users.

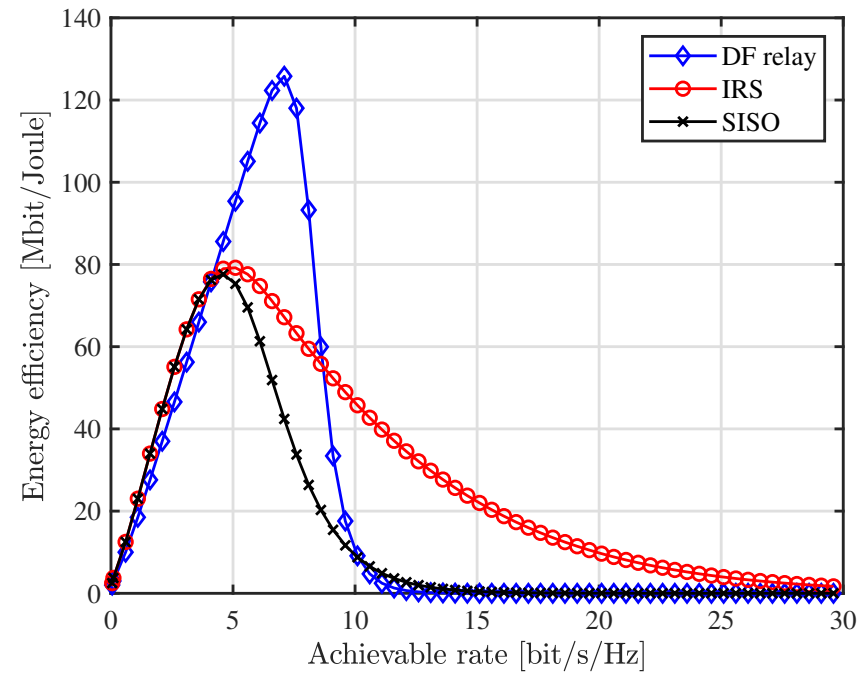

(b) $K=100$ users.

Figure 9. The energy efficiency based on the achievable rate $\bar{R}$ with a different number of users.

Finally, we study the IRS performance in the case of achievable rate and energy efficiency with respect to the transmit power as shown in Figure 10. In this simulation, we assign the number of users, $K=10$ users, and the power dissipation in each elements of IRS as $P_{\mathrm{e}}=5 \mathrm{~mW}$. We set a different defined number of IRS elements instead of the optimal number of elements that we applied in Figure 9. From Figure 10a, it is known that the achievable rate of IRS will be enhanced as long as the number of IRS elements and the transmit power increase as we obtained based on Equation (6). Figure 10b indicates that the IRS with the lowest number of elements attained the highest energy efficiency. The higher number of IRS elements increased the power dissipation in each element as defined in Equation (20), and thus the energy efficiency suffered. 


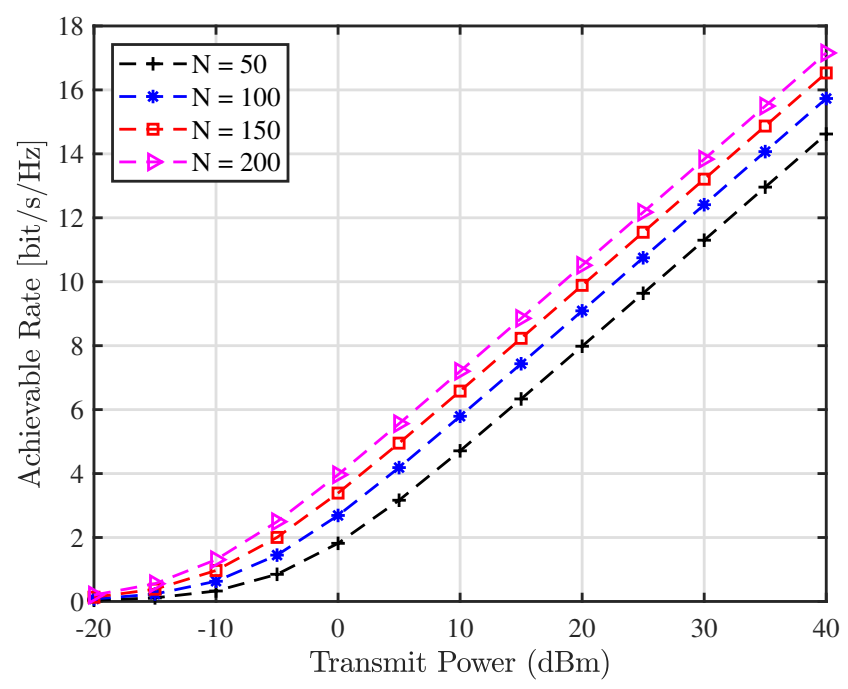

(a) The achievable rate based on the transmit power.

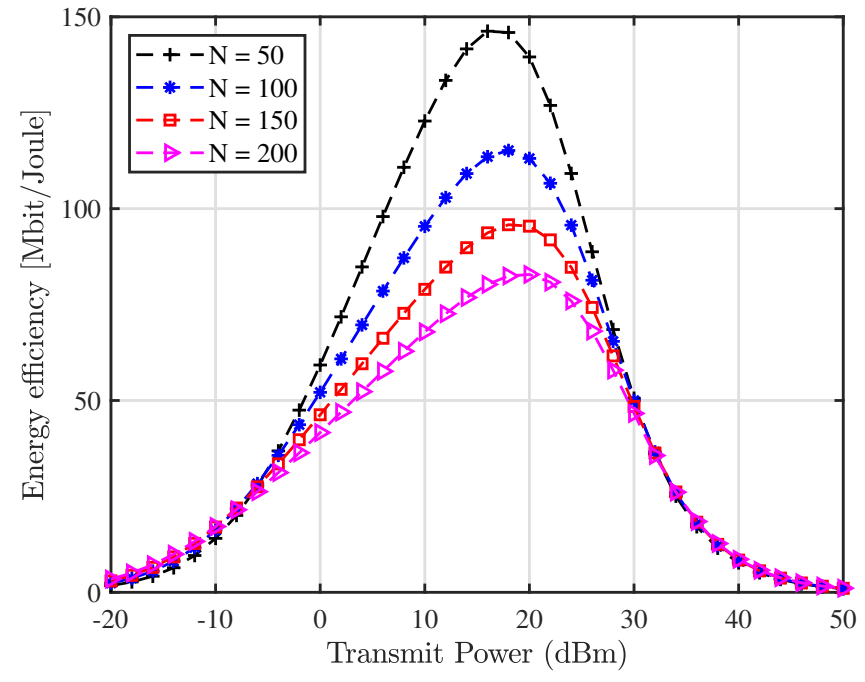

(b) The energy efficiency based on the transmit power.

Figure 10. The achievable rate and the energy efficiency based on the transmit power with different IRS elements.

\section{Conclusions}

We made a performance comparison among IRS-aided MU communication, DF relay aided MU communication, and basic MU communication in terms of the achievable rate, transmit power, and energy efficiency. We observed that, at best, a DF-relay-aided communication with a perfect CSI could match the performance of the IRS-aided network with an imperfect CSI when the channel estimation error was high and the number of users was large. We also observed that IRS-aided communication outright outperformed the DF relay case in a network with high transmit power and a lower number of users. Lastly, when the QoS requirements were high, the IRS-aided communication showed better energy efficiency compared with the other two scenarios.

Author Contributions: Conceptualization, M.M. and K.S.; methodology, M.M. and K.S.; software, M.M.; validation, K.S., S.B. and W.-J.H.; formal analysis, K.S., S.B. and W.-J.H.; investigation, M.M., K.S. and S.B.; resources, M.M. and K.S.; data curation, M.M.; writing-original draft preparation, M.M.; writing—review and editing, K.S. and S.B.; visualization, M.M.; supervision, K.S. and W.-J.H.; 
project administration, K.S.; funding acquisition, K.S. All authors have read and agreed to the published version of the manuscript.

Funding: This work was supported by the Ministry of Science and Technology of Taiwan under Grant MOST 110-2221-E-110-020.

Conflicts of Interest: The authors declare no conflict of interest.

\section{Abbreviations}

The following abbreviations are used in this manuscript:

$\begin{array}{ll}\text { 3GPP } & \text { 3rd Generation Partnership Project } \\ \text { 5G } & \text { 5th Generation } \\ \text { BS } & \text { Base Station } \\ \text { CSI } & \text { Channel State Information } \\ \text { DF } & \text { Decode and Forward } \\ \text { EE } & \text { Energy Efficiency } \\ \text { FR } & \text { Frequency Ranges } \\ \text { GHz } & \text { Giga Hertz } \\ \text { ITU } & \text { International Telecommunciation Union } \\ \text { IRS } & \text { Intelligent Reconfigurable Surface } \\ \text { LoS } & \text { Line of Sight } \\ \text { MIMO } & \text { Multiple Input Multiple Output } \\ \text { MRC } & \text { Maximum Ration Combining } \\ \text { MU } & \text { Multi Users } \\ \text { NLOS } & \text { Non-Line of Sight } \\ \text { QoS } & \text { Quality of Services } \\ \text { ZB } & \text { Zetta Bytes }\end{array}$

\section{References}

1. Tariq, F.; Khandaker, M.R.A.; Wong, K.-K.; Imran, M.A.; Bennis, M.; Debbah, M. A Speculative Study on 6G. IEEE Wirel. Commun. 2020, 27, 118-125. [CrossRef]

2. Basar, E.; Renzo, M.D.; Rosny, J.D.; Debbah, M.; Alouini, M.-S.; Zhang, R. Wireless Communications Through Reconfigurable Intelligent Surfaces. IEEE Access 2019, 7, 116753-116773. [CrossRef]

3. Guo, H.; Liang, Y.; Chen, J.; Larsson, E.G. Weighted Sum-Rate Maximization for Reconfigurable Intelligent Surface Aided Wireless Networks. IEEE Trans. Wirel. Commun. 2020, 19, 3064-3076. [CrossRef]

4. $\quad$ Tang, W.; Chen, M.Z.; Chen, X.; Dai, J.Y.; Han, Y.; Di Renzo, M.; Zeng, Y.; Jin, S.; Cheng, Q.; Cui, T.J. Wireless Communications With Reconfigurable Intelligent Surface: Path Loss Modeling and Experimental Measurement. IEEE Trans. Wirel. Commun. 2021, 20, 421-439. [CrossRef]

5. $\quad$ ElMossallamy, M.A.; Zhang, H.; Song, L.; Seddik, K.G.; Han, Z.; Li, G.Y. Reconfigurable Intelligent Surfaces for Wireless Communications: Principles, Challenges, and Opportunities. IEEE Trans. Cogn. Commun. Netw. 2020, 6, 990-1002. [CrossRef]

6. Tan, X.; Sun, Z.; Jornet, J.M.; Pados, D. Increasing indoor spectrum sharing capacity using smart reflect-array. In Proceedings of the 2016 IEEE International Conference on Communications (ICC), Kuala Lumpur, Malaysia, 23-27 May 2016; pp. 1-6. [CrossRef]

7. Huang, C.; Zappone, A.; Debbah, M.; Yuen, C. Achievable Rate Maximization by Passive Intelligent Mirrors. In Proceedings of the 2018 IEEE International Conference on Acoustics, Speech and Signal Processing (ICASSP), Calgary, AB, Canada, 15-20 April 2018; pp. 3714-3718. [CrossRef]

8. Yu, X.; Xu, D.; Schober, R. Optimal Beamforming for MISO Communications via Intelligent Reflecting Surfaces. In Proceedings of the 2020 IEEE 21st International Workshop on Signal Processing Advances in Wireless Communications (SPAWC), Atlanta, GA, USA, 26-29 May 2020; pp. 1-5. [CrossRef]

9. Li, A.; Song, L.; Vucetic, B.; Li, Y. Interference Exploitation Precoding for Reconfigurable Intelligent Surface Aided Multi-User Communications With Direct Links. IEEE Wirel. Commun. Lett. 2020, 9, 1937-1941. [CrossRef]

10. Zhang, H.; Di, B.; Song, L.; Han, Z. Reconfigurable Intelligent Surfaces Assisted Communications With Limited Phase Shifts: How Many Phase Shifts Are Enough? IEEE Trans. Veh. Tech. 2020, 69, 4498-4502. [CrossRef]

11. Di, B.; Zhang, H.; Song, L.; Li, Y.; Han, Z.; Poor, H.V. Hybrid Beamforming for Reconfigurable Intelligent Surface based Multi-User Communications: Achievable Rates With Limited Discrete Phase Shifts. IEEE J. Sel. Areas Commun. 2020, 38, 1809-1822. [CrossRef]

12. Di Renzo, M.; Ntontin, K.; Song, J.; Danufane, F.H.; Qian, X.; Lazarakis, F.; De Rosny, J.; Phan-Huy, D.T.; Simeone, O.; Zhang, R.; et al. Reconfigurable Intelligent Surfaces vs. Relaying: Differences, Similarities, and Performance Comparison. IEEE Open J. Commun. Soc. 2020, 1, 798-807. [CrossRef] 
13. Chatzigeorgiou, I. The Impact of 5G Channel Models on the Performance of Intelligent Reflecting Surfaces and Decode-andForward Relaying. In Proceedings of the 2020 IEEE 31st Annual International Symposium on Personal, Indoor and Mobile Radio Communications, London, UK, 31 August-3 September 2020; pp. 1-4. [CrossRef]

14. Björnson, E.; Ozdogan, O.; Larsson, E.G. Intelligent Reflecting Surface Versus Decode-and-Forward: How Large Surfaces are Needed to Beat Relaying? IEEE Wirel. Commun. Lett. 2020, 9, 244-248. [CrossRef]

15. Huang, C.; Zappone, A.; Alexandropoulos, G.C.; Debbah, M.; Yuen, C. Reconfigurable Intelligent Surfaces for Energy Efficiency in Wireless Communication. IEEE Trans. Wirel. Commun. 2019, 18, 4157-4170. [CrossRef]

16. Huang, C.; Alexandropoulos, G.C.; Zappone, A.; Debbah, M.; Yuen, C. Energy Efficient Multi-User MISO Communication Using Low Resolution Large Intelligent Surfaces. In Proceedings of the 2018 IEEE Globecom Workshops (GC Wkshps), Abu Dhabi, United Arab Emirates, 9-13 December 2018; pp. 1-6. [CrossReff]

17. 5G. Study on Channel Model for Frequencies from 0.5 to $100 \mathrm{GHz}$. 3GPP TR 38.901 (Release 14). 2018. Available online: https:/ / www.etsi.org/deliver/etsi_tr/138900_138999/138901/14.00.00_60/tr_138901v140000p.pdf (accessed on 16 June 2021).

18. Wu, Q.; Zhang, R. Intelligent Reflecting Surface Enhanced Wireless Network via Joint Active and Passive Beamforming. IEEE Trans. Wirel. Commun. 2019, 18, 5394-5409. [CrossRef]

19. Nadeem, Q.-U.-A.; Kammoun, A.; Chaaban, A.; Debbah, M.; Alouini, M.-S. Intelligent reflecting surface assisted wireless communication: Modeling and channel estimation. arXiv 2019, arXiv:1906.02360

20. He, Z.; Yuan, X. Cascaded Channel Estimation for Large Intelligent Metasurface Assisted Massive MIMO. IEEE Wirel. Commun. Lett. 2020, 9, 210-214. [CrossRef]

21. Taha, A.; Alrabeiah, M.; Alkhateeb, A. Enabling Large Intelligent Surfaces With Compressive Sensing and Deep Learning. IEEE Access 2021, 9, 44304-44321. [CrossRef]

22. Wang, Z.; Liu L.; Cui, S. Channel Estimation for Intelligent Reflecting Surface Assisted Multiuser Communications: Framework, Algorithms, and Analysis. IEEE Trans. Wirel. Commun. 2020, 19, 6607-6620. [CrossRef]

23. Dhok, S.; Raut, P.; Sharma, P.K.; Singh, K.; Li, C.-P. Non-Linear Energy Harvesting in RIS-assisted URLLC Networks for Industry Automation. IEEE Trans. Commun. 2021. [CrossRef]

24. Rappaport, T.S.; Xing, Y.; MacCartney, G.R.; Molisch, A.F.; Mellios, E.; Zhang, J. Overview of Millimeter Wave Communications for Fifth-Generation (5G) Wireless Networks-With a Focus on Propagation Models. IEEE Trans. Antennas Propag. 2017, 65, 6213-6230. [CrossRef]

25. Ma, B.; Zhang, H.; Zhang, Z. Joint power allocation and mode selection for D2D communications with imperfect CSI. China Commun. 2015, 12, 73-81. [CrossRef] 